\title{
A triumph of sensitivity
}

\author{
The Nobel Prize-winning observation of gravitational waves has required laser interferometry to be pushed to \\ extreme limits of sensitivity.
}

T he award of the 2017 Nobel Prize in Physics to Rainer Weiss, Barry Barish and Kip Thorne for the direct detection of gravitational waves in autumn 2015 is ultimately a triumph in the astonishing capabilities of optical science (https://www.nobelprize.org/nobel_prizes/ physics/laureates/2017/).

In particular, it is underpinned by decades of pain-staking engineering and ingenuity to push laser interferometry to unprecedented and mind-boggling levels of sensitivity and precision. The upgraded advanced Laser Interferometer Gravitational-Wave Observatory (aLIGO) in the US (www.ligo.org) that was responsible for the Nobel Prizewinning observations is the largest and most sensitive interferometer that has ever been built. It comprises two identical giant L-shaped Michelson interferometers (4-km-long arms) located in Hanford and Livingston that are capable of measuring a strain on the order $10^{-23}$ at a frequency window around $100 \mathrm{~Hz}$. The change in arm length in the interferometer when a gravitational wave passes through the instrument is on order of just $10^{-19} \mathrm{~m}$ (10,000 times smaller than a proton).

The engineering required to make measurements on this scale possible has placed stringent demands on all of aLIGO's optical parts. The mirrors (also called test masses) located at each end of the interferometer's arms have a mass of $40 \mathrm{~kg}$ and are made of ultrahigh-purity silica glass. They are coated with multiple layers of doped tantala and silica to provide almost perfect reflectivity at aLIGO's operational wavelength of 1,064 nm. The mirrors scatter less than $10 \mathrm{ppm}$ of the incident light, necessitating sub-atomic-level surface roughness. As part of a multi-staged sophisticated vibration isolation scheme designed to protect the interferometer from seismic disturbances, the mirrors are suspended using a series of thin $(400 \mu \mathrm{m}$ diameter) silica fibres and linked to a $360 \mathrm{~kg}$ quadruple pendulum mass system. Ultimately, the mirrors are so well protected from vibration that the random motion of the atoms within the mirrors and their housings can be detected.

The entire beam path of the interferometer is maintained under ultrahigh-vacuum conditions $\left(10^{-9}\right.$ torr $)$ to prevent air currents, dust and small temperature changes from disrupting the propagation of the laser beams. It takes 40 days of constant pumping to remove all the air from the system.

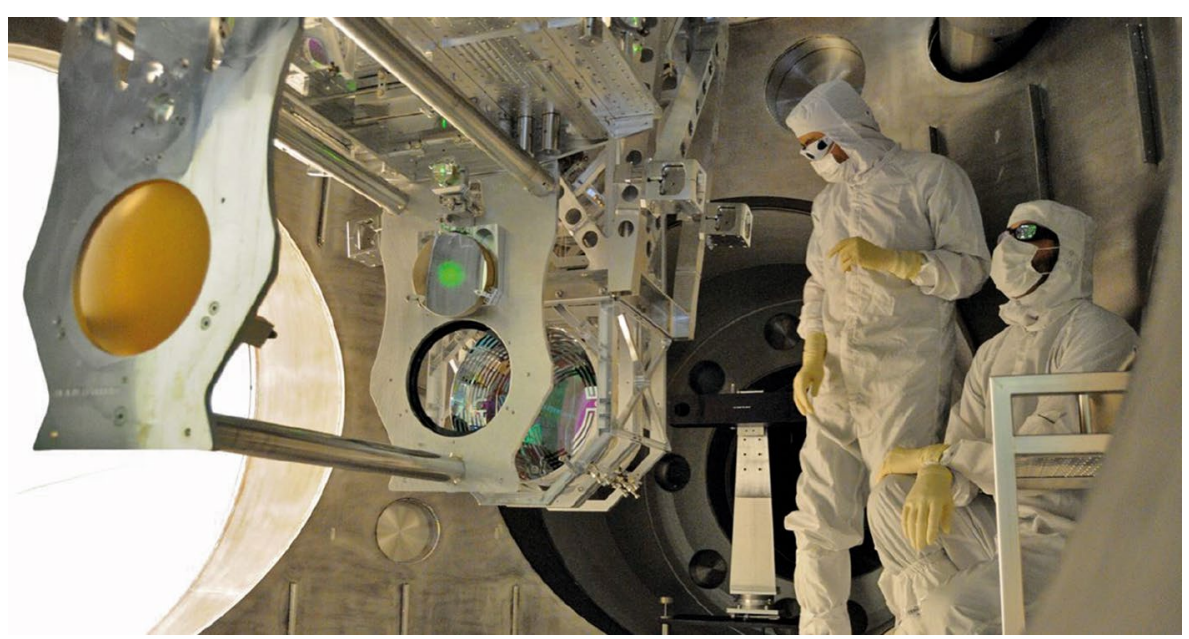

Credit: Caltech/MIT/LIGO Lab

The laser beam needed for aLIGO is based on a pre-stabilized laser system built by the Albert Einstein Institute and Laser Zentrum Hannover in Germany. It consists of multiple stages of power amplification and stabilization to deliver an $\sim 200 \mathrm{~W}$ beam of 1,064 $\mathrm{nm}$ laser light that is stable in amplitude at the parts per billion level. The beam originates from a custom-designed nonplanar ring oscillator that uses a specially shaped Nd:YAG crystal as a laser medium and resonator to generate a 1,064 $\mathrm{nm}$ seed beam with a power of $2 \mathrm{~W}$ when it is pumped by an $808 \mathrm{~nm}$ laser diode. The seed beam is then passed through a master oscillator power amplifier that features four amplifier rods in series to boost the beam power to $35 \mathrm{~W}$. Finally, a high-power oscillator is used to amplify the beam to its final power of around $200 \mathrm{~W}$. The beam's wavelength and amplitude are stabilized using a reference cavity and feedback control, before being injected into the interferometer.

While the achievements of aLIGO are impressive, even grander plans for optical detection of gravitational waves are on the horizon. In June 2017, following successful completion of a pathfinder project, the European Space Agency formally announced that it was proceeding with its Laser Interferometer Space Antenna (LISA) project (http://sci.esa.int/lisa/) that aims to create a giant laser interfometer in space using three spacecraft to create a beam path in the shape of an equilaterial triangle with 2.5-million-km-long arms. Each craft will feature two test-mass mirrors and two lasers pointing at the other craft to form a system of Michelson interferometers. The orbital motion of the triangle will allow LISA to not only detect the occurrence of a gravitational wave event but also its location. LISA will operate at a lower frequency range than aLIGO allowing it to detect galactic sources of gravitational waves. The plan is for LISA to be operational in 2034.

In a similar project, Japan is also planning a space-based interferometer called DECIGO (DECi-hertz Interferometer Gravitational wave Observatory; http:// tamago.mtk.nao.ac.jp/decigo/index_E.html) that again makes use of three spacecraft but with much shorter separations of $1,000 \mathrm{~km}$ to allow operation in the $0.1-10 \mathrm{~Hz}$ frequency band, between LISA and aLIGO.

As a final note, this year is the sixth time in the last decade that photonics has underpinned a Nobel Prize in Physics or Chemistry. Previous prizes with a strong connection to optics include the discovery of green fluorescent protein (2008), and the invention of low-loss optical fibres and the CCD image sensor (2009), particle manipulation in quantum optics (2012), the blue GaN light-emitting diode (2014) and super-resolution fluorescence microscopy (2014).

Published online: 31 October 2017 https://doi.org/10.1038/s41566-017-0046-7 